\title{
Correction to: Basic psychological need satisfaction across the retirement transition: Changes and longitudinal associations with depressive symptoms
}

\author{
Andreas Stenling ${ }^{1}\left(\mathbb{D} \cdot\right.$ Georg Henning ${ }^{2} \cdot$ Pär Bjälkebring $^{3,4} \cdot$ Susanne Tafvelin ${ }^{1} \cdot$ Marie Kivi $^{3} \cdot$ Boo Johansson $^{3}$. \\ Magnus Lindwall ${ }^{3,5}$
}

Published online: 31 December 2020

(c) Springer Science+Business Media, LLC, part of Springer Nature 2020

\section{Correction to: Motivation and Emotion https://doi.org/10.1007/s11031-020-09854-2}

The original version of this article unfortunately contained a mistake.

(a) The footnote text overlapped with the article text. However, it is now correct and given below:

We combined the retired $\mathrm{W} 2$ and retired $\mathrm{W} 3$ groups as their levels of and changes in need satisfaction and their effects on the second-order intercept and slope factors were identical. Hence, we reduced the dummy coded predictors to two instead of three to simplify the model.

(b) The alignment of Tables 1, 2, 3 and 5 are corrected. The updated tables are given below.

(c) In the Table 2, the heading "Comptence" should read as "Competence".

The original article has been corrected.

The original article can be found online at https://doi.org/10.1007/ s11031-020-09854-2.

Andreas Stenling

Andreas.stenling@umu.se

1 Department of Psychology, Umeå University, SE-901 87 Umeå, Sweden

2 German Centre of Gerontology, Berlin, Germany

3 Department of Psychology \& AgeCap, University of Gothenburg, Gothenburg, Sweden

4 Department of Psychology, The Ohio State University, Columbus, OH, USA

5 The Swedish School of Sport and Health Sciences, Stockholm, Sweden 
Table 1 Longitudinal measurement invariance across time and groups in the multiitem scales (BPNSFS and CES-D 8) and model fit of the LGCMs

\begin{tabular}{|c|c|c|c|c|c|c|c|}
\hline Model & $\chi^{2}$ & $d f$ & $p$ & RMSEA [90\% CI] & CFI & TLI & SRMR \\
\hline \multicolumn{8}{|l|}{ Autonomy } \\
\hline Configural & 72.360 & 60 & .1316 & $.018[.000, .031]$ & .998 & .994 & .018 \\
\hline Metric within groups & 81.598 & 76 & .3095 & $.011[.000, .025]$ & .999 & .998 & .025 \\
\hline Metric within and between groups & 84.953 & 82 & .3898 & $.007[.000, .023]$ & .999 & .999 & .025 \\
\hline Scalar within groups & 126.369 & 98 & .0284 & $.021[.007, .031]$ & .995 & .992 & .028 \\
\hline Scalar within and between groups & 148.067 & 104 & .0030 & $.025[.015, .034]$ & .992 & .989 & .033 \\
\hline Second-order LGCM & 206.031 & 110 & $<.001$ & $.036[.029, .044]$ & .982 & .977 & .045 \\
\hline \multicolumn{8}{|l|}{ Competence } \\
\hline Configural & 83.223 & 60 & .0253 & $.024[.009, .036]$ & .995 & .988 & .020 \\
\hline Metric within groups & 97.613 & 76 & .0482 & $.021[.002, .032]$ & .995 & .991 & .028 \\
\hline Metric within and between groups & 104.299 & 82 & .0489 & $.020[.002, .031]$ & .995 & .992 & .032 \\
\hline Scalar within groups & 147.156 & 98 & .0010 & $.028[.018, .036]$ & .990 & .985 & .033 \\
\hline Scalar within and between groups & 166.658 & 104 & $<.001$ & $.030[.021, .038]$ & .987 & .982 & .034 \\
\hline Second-order LGCM & 175.027 & 110 & $<.001$ & $.030[.021, .038]$ & .986 & .982 & .036 \\
\hline \multicolumn{8}{|l|}{ Relatedness } \\
\hline Configural & 70.216 & 60 & .1725 & $.016[.000, .030]$ & .998 & .995 & .021 \\
\hline Metric withi & 81.790 & 76 & .3043 & $.011[.000, .025]$ & .999 & .998 & .031 \\
\hline Metric within and betwee & 84.792 & 82 & .3945 & $.007[.000, .023]$ & .999 & .999 & .034 \\
\hline Scalar w & 108.662 & 98 & .2169 & $.013[.000, .025]$ & .998 & .997 & .037 \\
\hline Scalar within and betw & 113.618 & 104 & .2441 & $.012[.000, .024]$ & .998 & .997 & .040 \\
\hline Second-order LGCM & 137.191 & 112 & .0531 & $.018[.000, .028]$ & .994 & .993 & .046 \\
\hline \multicolumn{8}{|l|}{ Depressive symptoms } \\
\hline Configural & 2420.444 & 900 & $<.001$ & $.050[.048, .053]$ & .912 & .891 & .055 \\
\hline Metric within groups & 2448.966 & 956 & $<.001$ & $.049[.046, .051]$ & .913 & .900 & .057 \\
\hline Metric within and between groups & 2445.751 & 977 & $<.001$ & $.048[.045, .050]$ & .915 & .903 & .059 \\
\hline Scalar within groups & 2568.756 & 1033 & $<.001$ & $.047[.045, .050]$ & .911 & .904 & .059 \\
\hline Scalar within and between groups & 2645.825 & 1054 & $<.001$ & $.048[.045, .050]$ & .907 & .903 & .060 \\
\hline Second-order LGCM & 2634.837 & 1058 & $<.001$ & $.047[.045, .050]$ & .908 & .904 & .060 \\
\hline \multicolumn{8}{|l|}{ Multivariate LGCM } \\
\hline Autonomy - depressive symptoms & 6120.384 & 2963 & $<.001$ & $.040[.039, .041]$ & .895 & .888 & .059 \\
\hline Competence - depressive symptoms & 6023.328 & 2975 & $<.001$ & $.039[.038, .041]$ & .895 & .889 & .059 \\
\hline Relatedness - depressive symptoms & 5879.779 & 2971 & $<.001$ & $.038[.037, .040]$ & .900 & .894 & .063 \\
\hline
\end{tabular}




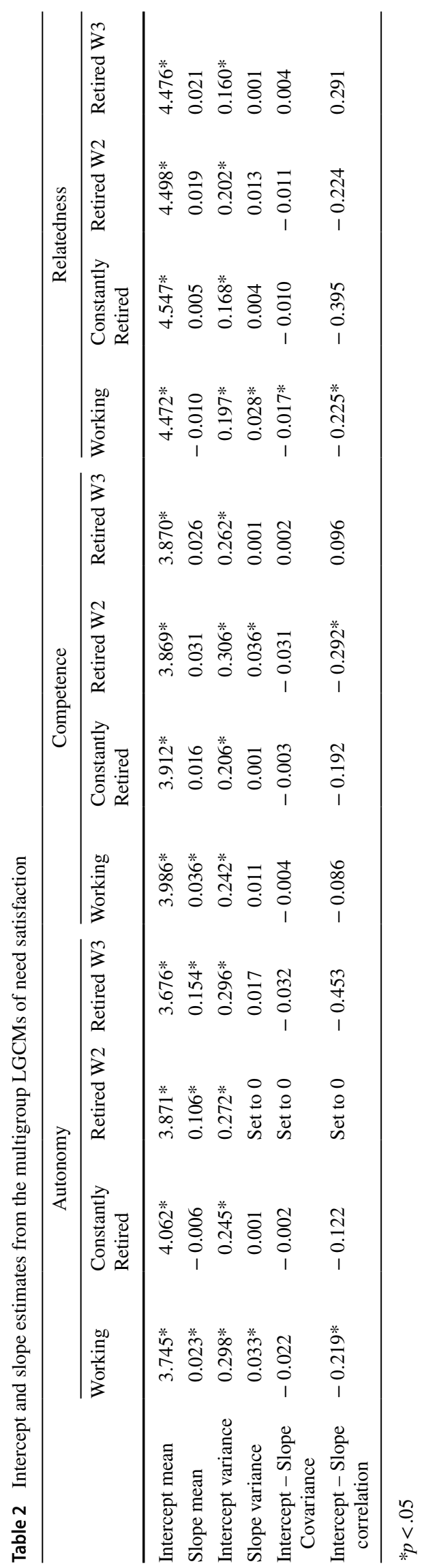

Table 3 Intercept and slope estimates from the multigroup LGCMs of depressive symptoms

\begin{tabular}{lcllr}
\hline & Working & $\begin{array}{l}\text { Con- } \\
\text { stantly } \\
\text { Retired }\end{array}$ & $\begin{array}{l}\text { Retired } \\
\text { W2 }\end{array}$ & \multicolumn{1}{l}{$\begin{array}{l}\text { Retired } \\
\text { W3 }\end{array}$} \\
\hline Intercept mean & $0.505^{*}$ & $0.427^{*}$ & $0.440^{*}$ & $0.526^{*}$ \\
Slope mean & -0.005 & 0.000 & 0.001 & $-0.035^{*}$ \\
Intercept variance & $0.131^{*}$ & $0.109^{*}$ & $0.132^{*}$ & $0.125^{*}$ \\
Slope variance & 0.001 & 0.001 & 0.014 & 0.001 \\
$\begin{array}{l}\text { Intercept-slope } \\
\text { covariance }\end{array}$ & -0.005 & 0.003 & -0.007 & -0.007 \\
$\begin{array}{l}\text { Intercept-slope } \\
\text { correlation }\end{array}$ & -0.451 & 0.270 & -0.162 & -0.571 \\
\hline$* p<.05$ & & & & \\
\hline
\end{tabular}


Table 5 Parameter estimates (unstandardized estimates) from the multivariate LGCMs

\begin{tabular}{lcccc}
\hline & Working & Retired & Retired W2 & Retired W3 \\
\hline$\alpha_{\text {autonomy }} \rightarrow \beta_{\text {depressive symptoms }}$ & -0.011 & -0.049 & -0.039 & -0.071 \\
$\alpha_{\text {depressive symptoms }} \rightarrow \beta_{\text {autonomy }}$ & -0.100 & -0.037 & -0.257 & -0.074 \\
$\alpha_{\text {autonomy }} \leftrightarrow \alpha_{\text {depressive symptoms }}$ & $-0.059^{*}$ & $-0.049^{*}$ & $-0.055^{*}$ & $-0.047^{*}$ \\
$\beta_{\text {depressive symptoms }} \leftrightarrow \beta_{\text {autonomy }}$ & -0.002 & $0^{\mathrm{a}}$ & $0^{\mathrm{a}}$ & $0^{\mathrm{a}}$ \\
$\mathrm{WP}_{\text {depressive symptoms } \leftrightarrow \mathrm{WP}_{\text {autonomy }}}$ & $-0.019^{*}$ & $-0.017^{*}$ & $-0.018^{*}$ & $-0.039^{*}$ \\
$\alpha_{\text {competence }} \rightarrow \beta_{\text {depressive symptoms }}$ & 0.004 & -0.011 & 0.004 & 0.056 \\
$\alpha_{\text {depressive symptoms }} \rightarrow \beta_{\text {competence }}$ & 0.007 & 0.029 & -0.019 & -0.017 \\
$\alpha_{\text {competence }} \leftrightarrow \alpha_{\text {depressive symptoms }}$ & $-0.052^{*}$ & $-0.039^{*}$ & $-0.052^{*}$ & $-0.071^{*}$ \\
$\beta_{\text {depressive symptoms }} \leftrightarrow \beta_{\text {competence }}$ & $0^{\mathrm{a}}$ & $0^{\mathrm{a}}$ & $0^{\mathrm{a}}$ & $0^{\mathrm{a}}$ \\
$\mathrm{WP}_{\text {depressive symptoms } \leftrightarrow \mathrm{WP}_{\text {competence }}}$ & $0.012^{*}$ & $-0.015^{*}$ & $-0.022^{*}$ & $-0.030^{*}$ \\
$\alpha_{\text {relatedness }} \rightarrow \beta_{\text {depressive symptoms }}$ & -0.012 & -0.036 & 0.058 & -0.015 \\
$\alpha_{\text {depressive symptoms }} \rightarrow \beta_{\text {relatedness }}$ & $-0.085^{*}$ & -0.033 & -0.068 & -0.052 \\
$\alpha_{\text {relatedness }} \leftrightarrow \alpha_{\text {depressive symptoms }}$ & $-0.042^{*}$ & $-0.034^{*}$ & $-0.038^{*}$ & $-0.029^{*}$ \\
$\beta_{\text {depressive symptoms }} \leftrightarrow \beta_{\text {relatedness }}$ & $0^{\mathrm{a}}$ & $0^{\mathrm{a}}$ & -0.002 & $0^{\mathrm{a}}$ \\
$\mathrm{WP}_{\text {depressive symptoms }} \leftrightarrow \mathrm{WP}_{\text {relatedness }}$ & $-0.012^{*}$ & $-0.011^{*}$ & -0.006 & $-0.016^{*}$ \\
\hline
\end{tabular}

Control variables in all of these models were age, years of education, sex, civil status, leisure activities, self-rated health, income satisfaction, and control over retirement (not in the working group)

$\alpha$ intercept, $\beta$ slope, $W P$ within-person

${ }^{\mathrm{a}}$ variances and covariances set to 0 to avoid inadmissible solutions

$* p<.05$

Publisher's Note Springer Nature remains neutral with regard to jurisdictional claims in published maps and institutional affiliations. 